\title{
Optimal Multi-Channel Cooperative Sensing in Cognitive Radio Networks
}

\author{
Rongfei Fan and Hai Jiang, Member, IEEE
}

\begin{abstract}
In this paper, optimal multi-channel cooperative sensing strategies in cognitive radio networks are investigated. A cognitive radio network with multiple potential channels is considered. Secondary users cooperatively sense the channels and send the sensing results to a coordinator, in which energy detection with a soft decision rule is employed to estimate whether there are primary activities in the channels. An optimization problem is formulated, which maximizes the throughput of secondary users while keeping detection probability for each channel above a pre-defined threshold. In particular, two sensing modes are investigated: slotted-time sensing mode and continuous-time sensing mode. With a slotted-time sensing mode, the sensing time of each secondary user consists of a number of minislots, each of which can be used to sense one channel. The initial optimization problem is shown to be a nonconvex mixedinteger problem. A polynomial-complexity algorithm is proposed to solve the problem optimally. With a continuous-time sensing mode, the sensing time of each secondary user for a channel can be any arbitrary continuous value. The initial nonconvex problem is converted into a convex bilevel problem, which can be successfully solved by existing methods. Numerical results are presented to demonstrate the effectiveness of our proposed algorithms.
\end{abstract}

Index Terms-Cognitive radio, spectrum sensing, throughput maximization.

\section{INTRODUCTION}

D UE to the rapid growth of wireless communications in recent years, so far almost all the appropriate spectrum has been allocated for various wireless applications in different regions. So a spectrum scarcity problem is expected for the development of new wireless applications in the near future. On the other hand, severe under-utilization of licensed spectrum at a time or a location has been observed by measurements of wireless spectrum usage [1] [2]. This has motivated the idea of opportunistic spectrum access, which means that unlicensed (secondary) users can utilize the spectrum when and where the licensed (primary) users are not using it. As an emerging technique to realize this idea, cognitive radio has received much attention recently, in which the secondary users are able to sense the spectrum, analyze the spectrum statistics, and adjust their transmissions according to the time-varying environment [3].

Manuscript received March 31, 2009; revised October 17, 2009 and January 7, 2010; accepted January 13, 2010. The associate editor coordinating the review of this paper and approving it for publication was R. Nabar.

The authors are with the Department of Electrical and Computer Engineering, University of Alberta, Edmonton, Alberta, Canada T6G 2V4 (e-mail: rongfei@ualberta.ca, hai.jiang@ece.ualberta.ca).

This work was supported by an Alberta Ingenuity New Faculty Award from the Alberta Innovates - Technology Futures, Alberta, Canada.

Digital Object Identifier 10.1109/TWC.2010.03.090467
Before accessing the spectrum, secondary users need to check (by spectrum sensing) whether primary users are active. If primary users are using the spectrum, the secondary users should defer their transmissions. The performance of the spectrum sensing can be measured by two parameters: detection probability (i.e., the probability that, if there are primary activities, the secondary users can detect them successfully) and false alarm probability (i.e., the probability that, if there are no primary activities, the secondary users falsely estimate that primary users are active) [4]. Apparently, a higher detection probability can give primary users more protection, while a higher false alarm probability will lead to more waste of the spectral opportunities. To improve the accuracy of spectrum sensing in a fading environment, cooperative spectrum sensing [5][6][7] can be adopted. Multiple secondary users sense the spectrum independently, and send the results to a fusion center, which will make the final estimation on whether there are primary activities in the channel.

For spectrum sensing, a slotted time frame structure is widely used [8]-[11]. In each time slot, the first portion is used for spectrum sensing, and the second portion is used for packet transmission (if the channel is detected idle). A longer sensing time in a time slot will lead to a higher detection probability and a lower false alarm probability, which are desired. But it also results in less time in actual information transmission (assuming the duration of each time slot is fixed). Therefore, a tradeoff exists in the sensing time setting. The optimal tradeoff in sensing time setting is investigated in [12][13] so as to optimally utilize the transmission opportunities in a single channel. Different from the work for a single channel, in this paper, we investigate the sensing time setting for a multi-user multi-channel case with cooperative sensing. The sensing time setting determines the length of the sensing time in a slot and how the total sensing time (of the multiple users) in a slot is distributed among all the channels. Optimization problems are formulated to maximize the throughput of secondary users. We prove that, although the initial optimization problems are nonconvex and are with NP-hard complexity to be solved directly, they can be successfully converted into convex mixedinteger subproblems or a convex bilevel problem, which are ready to be solved by existing methods.

The rest of the paper is organized as follows. The system model is given in Section II. The problem of optimal sensing time setting is formulated and solved in Section III when the sensing time for each channel is a number of mini-slots, and in Section IV when the sensing time for each channel is a continuous variable. Numerical results are presented in Section V. Finally concluding remarks are given in Section VI. 


\section{System Model}

We consider a cognitive radio network with $N$ frequency bands (termed channels in the sequel) and $M$ secondary users. In each channel, a primary user exists (which may not be active all the time). There is a coordinator in the cognitive radio network, which is responsible to collect sensing results from the secondary users, and estimate the busy/idle status of each channel. The coordinator also assigns a secondary user to each channel for information transmission. If the coordinator estimates a channel, say channel $n$, to be idle, it notifies the secondary user assigned in the channel to transmit. The transmission power is $P_{n}^{s}$, and the transmission rate is given by $\log \left(1+S N R_{n}^{s s}\right)$, where $S N R_{n}^{s s}$ means the signal-to-noise ratio (SNR) from the secondary user to its receiver at channel $n$.

A synchronous system is assumed, and time is divided into fixed-length slots. In each slot, the primary user in a channel is either active for the whole slot, or idle for the whole slot. Each slot is further partitioned into two phases: sensing phase and transmission phase. The duration of the sensing phase is a design parameter. In the sensing phase, a secondary user can sense a number of channels sequentially by energy detection, and the sampling rate of the received signal in a channel is $\mu$. The transmission phase is used for the secondary users assigned to the channels to transmit, if the channels are estimated to be idle. It is assumed that the channel gains in each channel (from the primary user to secondary users or between secondary users) keep fixed within the duration of a time slot.

Let $t_{n}^{m}$ denote the time duration that secondary user $m$ spends in sampling channel $n$. So within duration $t_{n}^{m}$, user $m$ has $\mu t_{n}^{m}$ samples for channel $n$, which follow a binary hypothesis:

$$
\begin{array}{lll}
\mathcal{H}_{n}^{0}: & y_{n}^{m}(i)=w_{n}(i), & i=1,2, \ldots, \mu t_{n}^{m} \\
\mathcal{H}_{n}^{1}: & y_{n}^{m}(i)=r_{n}^{m}(i)+w_{n}(i), & i=1,2, \ldots, \mu t_{n}^{m}
\end{array}
$$

where $\mathcal{H}_{n}^{0}$ and $\mathcal{H}_{n}^{1}$ mean that the primary user in channel $n$ is idle and busy respectively, $i$ is the sample index, $y_{n}^{m}(\cdot)$ is the received signal of channel $n$ at secondary user $m$, $w_{n}(\cdot)$ is background noise in channel $n$, which is assumed to be circular symmetric complex Gaussian (CSCG) with mean being zero and variance being $\sigma^{2}$, and $r_{n}^{m}(\cdot)$ is the signal of primary user in channel $n$ received at secondary user $m$. Similar to [12], $r_{n}^{m}(i)$ is assumed to be a zero mean CSCG random variable. Furthermore, we also suppose $r_{n}^{m}(i)$ 's are independent and identically distributed random variables with variance $\left(\sigma_{n}^{r}\right)^{2}$ for $m=1,2, \ldots, M$. This assumption is valid for a small-sized cognitive network (i.e., distance between the secondary users is much less than the distance from the primary user to the secondary users).

Then, the test statistic of secondary user $m$ 's received signal energy in channel $n$ is calculated as

$$
T_{n}^{m}(y)=\frac{1}{\mu t_{n}^{m}} \sum_{i=1}^{\mu t_{n}^{m}}\left|y_{n}^{m}(i)\right|^{2} .
$$

The test statistic by secondary user $m$ for channel $n$ is sent to the coordinator, which collects all values of $T_{n}^{m}(y)$ 's from all the secondary users. Then the overall test statistic for channel $n$ is calculated at the coordinator as

$$
T_{n}^{\text {all }}(y)=\frac{\sum_{m=1}^{M} \mu t_{n}^{m} \cdot T_{n}^{m}(y)}{\sum_{m=1}^{M} \mu t_{n}^{m}} .
$$

The overall test statistic is compared with a threshold $\varepsilon_{n}$. The primary user in channel $n$ is estimated to be idle if $T_{n}^{\text {all }}(y) \leq \varepsilon_{n}$, or busy otherwise. This process is referred to as soft decisional cooperative spectrum sensing, and the detection probability and false alarm probability in the process are given [12] as

$$
\begin{aligned}
& P_{n}^{d}\left(\sum_{m=1}^{M} t_{n}^{m}, \varepsilon_{n}\right)=\operatorname{Pr}\left(T_{n}^{\text {all }}(y)>\varepsilon_{n} \mid \mathcal{H}_{n}^{1}\right) \\
& \quad=Q\left(\left(\frac{\varepsilon_{n}}{\sigma^{2}}-\gamma_{n}-1\right) \sqrt{\mu \sum_{m=1}^{M} t_{n}^{m} /\left(\gamma_{n}+1\right)^{2}}\right)
\end{aligned}
$$

and

$$
\begin{aligned}
P_{n}^{f}\left(\sum_{m=1}^{M} t_{n}^{m}, \varepsilon_{n}\right) & =\operatorname{Pr}\left(T_{n}^{\text {all }}(y)>\varepsilon_{n} \mid \mathcal{H}_{n}^{0}\right) \\
& =Q\left(\left(\frac{\varepsilon_{n}}{\sigma^{2}}-1\right) \sqrt{\mu \sum_{m=1}^{M} t_{n}^{m}}\right)
\end{aligned}
$$

respectively, where $\gamma_{n}$ is the average SNR of primary user signal received by a secondary user in channel $n$, defined as $\gamma_{n} \triangleq \frac{\left(\sigma_{n}^{r}\right)^{2}}{\sigma^{2}}$, and $Q(\cdot)$ is the $\mathrm{Q}$ function, defined as

$$
Q(x)=\frac{1}{\sqrt{2 \pi}} \int_{x}^{\infty} \exp \left(-\frac{z^{2}}{2}\right) d z .
$$

In a real system, the detection probability $P_{n}^{d}$ should be no less than 0.5 and the false alarm probability should be no larger than 0.5. From (4) and (5), the constraints $P_{n}^{d} \geq 0.5$ and $P_{n}^{f} \leq 0.5$ are equivalent to the following inequality.

$$
\sigma^{2} \leq \varepsilon_{n} \leq \sigma^{2}\left(1+\gamma_{n}\right), \quad n=1,2, \ldots, N .
$$

The following equation rewrites the false alarm probability as a function of the detection probability $P_{n}^{d}$.

$$
\begin{aligned}
& P_{n}^{f}\left(\sum_{m=1}^{M} t_{n}^{m}, P_{n}^{d}\right)= \\
& \quad Q\left(\left(\gamma_{n}+1\right) Q^{-1}\left(P_{n}^{d}\right)+\gamma_{n} \sqrt{\mu \sum_{m=1}^{M} t_{n}^{m}}\right) .
\end{aligned}
$$

Within this expression, the constraint $P_{n}^{f} \leq 0.5$ is equivalent to

$$
\left(\gamma_{n}+1\right) Q^{-1}\left(P_{n}^{d}\right)+\gamma_{n} \sqrt{\mu \sum_{m=1}^{M} t_{n}^{m}} \geq 0, \quad n=1,2, \ldots, N .
$$

From (4) and (5) it can be seen that only the total time used to sense channel $n$, i.e., $\sum_{m=1}^{M} t_{n}^{m}$, affects the detection performance for channel $n$, regardless of how this total time is distributed among the secondary users. 
Slot

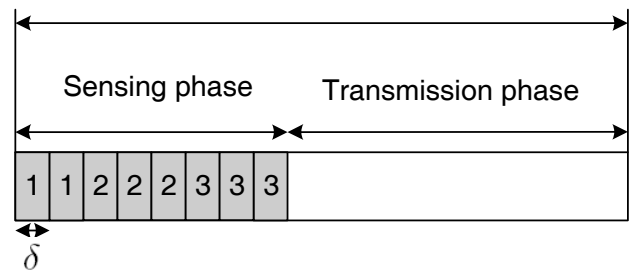

User 2

$$
\begin{array}{|l|l|l|l|l|l|l|l|l|}
\hline 2 & 2 & 2 & 2 & 3 & 3 & 3 & 1 \\
\hline
\end{array}
$$

User 3

\begin{tabular}{|l|l|l|l|l|l|l|l|l|}
\hline 1 & 1 & 1 & 2 & 2 & 3 & 3 & 3 & \\
\hline
\end{tabular}

Fig. 1. The slotted-time sensing mode with 3 channels and 3 secondary users.

For the sensing in channel $n$, we have the following four scenarios:

- If channel $n$ is idle and is estimated by the coordinator to be idle, then the secondary user assigned to channel $n$ will transmit in the associated transmission phase of the slot, with the average transmission rate given by $R_{n}^{0}=\mathbb{E}\left(\log \left(1+\frac{\left|h_{n}^{s s}\right|^{2} P_{n}^{s}}{\sigma^{2}}\right)\right)$, where $h_{n}^{s s}$ is the channel coefficient from the secondary user assigned to channel $n$ to its receiver, and $\mathbb{E}(\cdot)$ means expectation.

- If channel $n$ is idle and is estimated by the coordinator to be busy (i.e., a false alarm happens), the secondary user assigned to channel $n$ will not transmit in the associated transmission phase of the slot.

- If channel $n$ is busy and is estimated by the coordinator to be busy, the secondary user assigned to channel $n$ will not transmit in the associated transmission phase of the slot.

- If channel $n$ is busy and is estimated by the coordinator to be idle (i.e., a missed detection happens), then the secondary user assigned to channel $n$ will transmit in the associated transmission phase of the slot. As the primary user's signal will serve as an interference to the secondary transmission, the average transmission rate of the secondary user is given by $R_{n}^{1}=\mathbb{E}\left(\log \left(1+\frac{\left|h_{n}^{s s}\right|^{2} P_{n}^{s}}{\left|h_{n}^{p s}\right|^{2} P_{n}^{p}+\sigma^{2}}\right)\right)$, where $P_{n}^{p}$ is the transmission power of the primary user in channel $n$, and $h_{n}^{p s}$ is the channel coefficient from the primary user to the secondary receiver in channel $n$. It can be seen that $R_{n}^{0}>R_{n}^{1}$.

In the sensing phase, a secondary user needs to sequentially sense a number of channels, using one of the two modes: slotted-time sensing mode and continuous-time sensing mode.

- Slotted-time sensing mode: The sensing phase in each slot is further divided into a number, $k$, of mini-slots, each with duration $\delta$. Here the value of $k$ is a parameter to be optimized. Each mini-slot can be used by a secondary user to sense any channel. Fig. 1 shows an example for $N=3$ channels, $M=3$ secondary users, and $k=8$ mini-slots in a sensing phase. The number inside each mini-slot means the channel to be sensed. Note that in the example, user 2 first senses channel 2, then channel 3, and
Slot

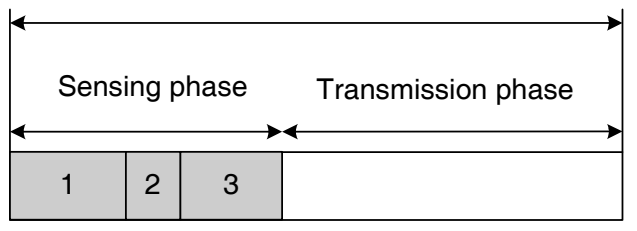

User 2

\begin{tabular}{|l|l|l|l|}
\hline 2 & 3 & 1 & \\
\hline
\end{tabular}

User 3

\begin{tabular}{|l|l|l|l|}
\hline 3 & 1 & 2 & \\
\hline
\end{tabular}

Fig. 2. The continuous-time sensing mode with 3 channels and 3 secondary users.

finally channel 1. Actually the sensing order of channels 1, 2 and 3 does not affect the detection performance.

- Continuous-time sensing mode: In the sensing phase, the time to sense a channel can be any arbitrary-length duration bounded by the total duration in the sensing phase, while the duration of the sensing phase is also a parameter to be optimized. Fig. 2 shows an example for $N=3$ channels and $M=3$ secondary users.

In the following two sections, optimal sensing time settings in the slotted-time sensing mode and continuous-time sensing mode are investigated respectively to maximize the total throughput of the secondary network.

\section{Optimal Sensing Time Setting in the Slotted-Time Sensing Mode}

In the system, the sensing phase in a slot has $k$ mini-slots. The value of $k$ is a parameter to be optimized. Each mini-slot can be used by a secondary user to sense a channel. So there are totally $k M$ mini-slots among the $M$ secondary users to sense the $N$ channels. Let $k_{n}>0$ denote the number of minislots (among the $k M$ mini-slots) that are used for sensing channel $n \in\{1,2, \ldots, N\}$. Then we have

$$
\sum_{n=1}^{N} k_{n}=k M
$$

Let $T$ denote the length of a time slot. Then the average throughput of channel $n$ can be expressed [12] as

$$
\begin{aligned}
C_{n}\left(k, k_{n}, \varepsilon_{n}\right)= & \frac{T-k \delta}{T}\left(\operatorname{Pr}\left(\mathcal{H}_{n}^{0}\right)\left(1-P_{n}^{f}\left(k_{n}, \varepsilon_{n}\right)\right) R_{n}^{0}\right. \\
& \left.+\operatorname{Pr}\left(\mathcal{H}_{n}^{1}\right)\left(1-P_{n}^{d}\left(k_{n}, \varepsilon_{n}\right)\right) R_{n}^{1}\right)
\end{aligned}
$$

where

$$
P_{n}^{d}\left(k_{n}, \varepsilon_{n}\right)=Q\left(\left(\frac{\varepsilon_{n}}{\sigma^{2}}-\gamma_{n}-1\right) \sqrt{\frac{k_{n} \delta \mu}{\left(\gamma_{n}+1\right)^{2}}}\right)
$$

and

$$
P_{n}^{f}\left(k_{n}, \varepsilon_{n}\right)=Q\left(\left(\frac{\varepsilon_{n}}{\sigma^{2}}-1\right) \sqrt{k_{n} \delta \mu}\right)
$$

are from (4) and (5), respectively, $\operatorname{Pr}\left(\mathcal{H}_{n}^{0}\right) \geq 0$ is the available probability of channel $n$, and $\operatorname{Pr}\left(\mathcal{H}_{n}^{1}\right)=1-\operatorname{Pr}\left(\mathcal{H}_{n}^{0}\right) \geq 0$ is the busy probability of channel $n$. 
Our goal is to maximize the throughput of secondary users in all the channels, denoted $C\left(k,\left\{k_{n}\right\},\left\{\varepsilon_{n}\right\}\right),{ }^{1}$ while keeping the detection probability of any channel, $P_{n}^{d}$, above a prespecified threshold $P_{t h}\left(P_{t h}>0.5\right)$ and the false alarm probability of any channel, $P_{n}^{f}$, no larger than 0.5 . So the problem can be defined as follows.

\section{Problem P1 :}

$$
\begin{array}{cl}
\max _{k,\left\{k_{n}\right\},\left\{\varepsilon_{n}\right\}} & C\left(k,\left\{k_{n}\right\},\left\{\varepsilon_{n}\right\}\right)=\frac{T-k \delta}{T} \\
& \quad \sum_{n=1}^{N}\left(\operatorname{Pr}\left(\mathcal{H}_{n}^{0}\right)\left(1-P_{n}^{f}\left(k_{n}, \varepsilon_{n}\right)\right) R_{n}^{0}\right. \\
& \left.\quad+\operatorname{Pr}\left(\mathcal{H}_{n}^{1}\right)\left(1-P_{n}^{d}\left(k_{n}, \varepsilon_{n}\right)\right) R_{n}^{1}\right) \\
\text { s.t. } & P_{n}^{d}\left(k_{n}, \varepsilon_{n}\right) \geq P_{t h}, \quad n=1,2, \ldots, N \\
& \sigma^{2} \leq \varepsilon_{n} \leq \sigma^{2}\left(1+\gamma_{n}\right), \quad n=1,2, \ldots, N \\
& \sum_{n=1}^{N} k_{n}=k M \\
& k_{n}>0, k_{n} \in \mathcal{I}, \quad n=1,2, \ldots, N \\
& 0<k \leq\left\lfloor\frac{T}{\delta}\right\rfloor, \quad k \in \mathcal{I} .
\end{array}
$$

Here $\mathcal{I}$ is the set of all positive integers. Note that the constraints $\sigma^{2} \leq \varepsilon_{n} \leq \sigma^{2}\left(1+\gamma_{n}\right)$ are equivalent to the constraints $P_{n}^{d} \geq 0.5$ and $P_{n}^{f} \leq 0.5$ according to equations (11) and (12).

\section{A. Nonconvexity of Problem Pl}

Lemma 1: Problem P1 is not a convex problem.

Proof: We use proof by contradiction. Assume that problem $\mathrm{P} 1$ is a convex problem. Thus, the objective function $C\left(k,\left\{k_{n}\right\},\left\{\varepsilon_{n}\right\}\right)$ is a concave function with respect to $k,\left\{k_{n}\right\}$, and $\left\{\varepsilon_{n}\right\}$. Based on (11) and (12), we have

$$
\begin{aligned}
\frac{\partial^{2} P_{n}^{f}\left(k_{n}, \varepsilon_{n}\right)}{\partial k_{n}{ }^{2}} & =\frac{1}{4 \sqrt{2 \pi}}\left(\frac{\varepsilon_{n}}{\sigma^{2}}-1\right) e^{-\frac{\left(\left(\frac{\varepsilon_{n}}{\sigma^{2}}-1\right) \sqrt{k_{n} \delta \mu}\right)^{2}}{2}} \\
& \cdot\left(\sqrt{\frac{\delta \mu}{k_{n}^{3}}}+(\delta \mu)^{3 / 2}\left(\frac{\varepsilon_{n}}{\sigma^{2}}-1\right)^{2} \frac{1}{\sqrt{k_{n}}}\right)
\end{aligned}
$$

and

$$
\begin{gathered}
\frac{\partial^{2} P_{n}^{d}\left(k_{n}, \varepsilon_{n}\right)}{\partial k_{n}^{2}}= \\
\frac{1}{4 \sqrt{2 \pi}}\left(\frac{\varepsilon_{n}}{\sigma^{2}}-\gamma_{n}-1\right) e^{-\frac{\left(\left(\frac{\varepsilon_{n}}{\sigma^{2}}-\gamma_{n}-1\right) \frac{\sqrt{k_{n} \delta \mu}}{\left(\gamma_{n}+1\right)}\right)^{2}}{2}} \\
\cdot\left(\sqrt{\frac{\delta \mu}{\left(\gamma_{n}+1\right)^{2}}} \frac{1}{\sqrt{k_{n}^{3}}}+\frac{(\delta \mu)^{3 / 2}}{\left(\gamma_{n}+1\right)^{3}}\left(\frac{\varepsilon_{n}}{\sigma^{2}}-\gamma_{n}-1\right)^{2} \frac{1}{\sqrt{k_{n}}}\right) .
\end{gathered}
$$

Among the constraints of problem P1, we have $\sigma^{2} \leq \varepsilon_{n} \leq$ $\sigma^{2}\left(1+\gamma_{n}\right)$. So in the feasible region of problem P1, we have

$$
\frac{\partial^{2} P_{n}^{f}\left(k_{n}, \varepsilon_{n}\right)}{\partial k_{n}^{2}}>0
$$

and

$$
\frac{\partial^{2} P_{n}^{d}\left(k_{n}, \varepsilon_{n}\right)}{\partial k_{n}^{2}}<0
$$

\footnotetext{
${ }^{1}$ Note that here $\left\{k_{n}\right\}$ means the set of $\left\{k_{1}, k_{2}, \ldots, k_{N}\right\}$, and $\left\{\varepsilon_{n}\right\}$ means the set of $\left\{\varepsilon_{1}, \varepsilon_{2}, \ldots, \varepsilon_{N}\right\}$.
}

Since $\operatorname{Pr}\left(\mathcal{H}_{n}^{1}\right)=1-\operatorname{Pr}\left(\mathcal{H}_{n}^{0}\right)$, it can be obtained that

$$
\begin{gathered}
\frac{\partial^{2} C\left(k,\left\{k_{n}\right\},\left\{\varepsilon_{n}\right\}\right)}{\partial k_{n}{ }^{2}}=\frac{T-k \delta}{T} \cdot\left(-\frac{\partial^{2} P_{n}^{d}\left(k_{n}, \varepsilon_{n}\right)}{\partial k_{n}{ }^{2}} R_{n}^{1}\right. \\
\left.-\operatorname{Pr}\left(\mathcal{H}_{n}^{0}\right)\left(\frac{\partial^{2} P_{n}^{f}\left(k_{n}, \varepsilon_{n}\right)}{\partial k_{n}{ }^{2}} R_{n}^{0}-\frac{\partial^{2} P_{n}^{d}\left(k_{n}, \varepsilon_{n}\right)}{\partial k_{n}{ }^{2}} R_{n}^{1}\right)\right) .
\end{gathered}
$$

Therefore, if

$$
\operatorname{Pr}\left(\mathcal{H}_{n}^{0}\right)<\frac{-\frac{\partial^{2} P_{n}^{d}\left(k_{n}, \varepsilon_{n}\right)}{\partial k_{n}{ }^{2}} R_{n}^{1}}{\frac{\partial^{2} P_{n}^{f}\left(k_{n}, \varepsilon_{n}\right)}{\partial k_{n}{ }^{2}} R_{n}^{0}-\frac{\partial^{2} P_{n}^{d}\left(k_{n}, \varepsilon_{n}\right)}{\partial k_{n}{ }^{2}} R_{n}^{1}}
$$

then we have

$$
\frac{\partial^{2} C\left(k,\left\{k_{n}\right\},\left\{\varepsilon_{n}\right\}\right)}{\partial k_{n}^{2}}>0
$$

which contradicts the assumption that $C\left(k,\left\{k_{n}\right\},\left\{\varepsilon_{n}\right\}\right)$ is a concave function with respect to variable $k_{n}$. Note that, from (15) and (16), it can be seen that the right-hand side of (17) is within $(0,1)$. Therefore, there exists $\operatorname{Pr}\left(\mathcal{H}_{n}^{0}\right)$ such that inequality (17) holds.

This completes the proof.

Besides being nonconvex, problem $\mathrm{P} 1$ is also a mixedinteger problem, which is usually NP-hard to be solved directly [14]. In order to solve problem P1, we resort to transformation of the problem into subproblems with low complexity, as follows.

In the expression of the objective function $C\left(k,\left\{k_{n}\right\},\left\{\varepsilon_{n}\right\}\right)$ in problem $\mathrm{P} 1$, it can be seen that variable $k$ appears only in the term $\frac{T-k \delta}{T}$. So problem P1 can be transformed to

$$
\begin{array}{cl}
\max _{k} & C(k)=\frac{T-k \delta}{T} \cdot U^{*}(k) \\
\text { s.t. } & 0<k \leq\left\lfloor\frac{T}{\delta}\right\rfloor, k \in \mathcal{I}
\end{array}
$$

where $U^{*}(k)$ is the optimal objective value of the following problem with a specific $k$ value:

Problem P2 (with a specific $k$ value)

$$
\begin{array}{cc}
\max _{\left\{k_{n}\right\},\left\{\varepsilon_{n}\right\}} & U\left(\left\{k_{n}\right\},\left\{\varepsilon_{n}\right\}\right)= \\
& \sum_{n=1}^{N}\left(\operatorname{Pr}\left(\mathcal{H}_{n}^{0}\right)\left(1-P_{n}^{f}\left(k_{n}, \varepsilon_{n}\right)\right) R_{n}^{0}\right. \\
& \left.\quad+\operatorname{Pr}\left(\mathcal{H}_{n}^{1}\right)\left(1-P_{n}^{d}\left(k_{n}, \varepsilon_{n}\right)\right) R_{n}^{1}\right) \\
\text { s.t. } \quad & P_{n}^{d}\left(k_{n}, \varepsilon_{n}\right) \geq P_{t h}, \quad n=1,2, \ldots, N \\
& \sigma^{2} \leq \varepsilon_{n} \leq \sigma^{2}\left(1+\gamma_{n}\right), \quad n=1,2, \ldots, N \\
& \sum_{n=1}^{N} k_{n}=k M \\
& k_{n}>0, k_{n} \in \mathcal{I}, \quad n=1,2, \ldots, N \\
& k \in \mathcal{I} .
\end{array}
$$

Problem P2 is actually a subproblem of problem P1. In the following, we first discuss the properties of problem P2 and provide an optimal solution to it in Section III-B, and then provide an optimal algorithm to solve problem P1 in Section III-C based on the optimal solution of problem P2.

\section{B. Properties and Optimal Solution of Problem P2}

Lemma 2: With the condition $P_{t h}>0.5$, the objective function $U\left(\left\{k_{n}\right\},\left\{\varepsilon_{n}\right\}\right)$ in problem P2 achieves the maximal value when $P_{n}^{d}\left(k_{n}, \varepsilon_{n}\right)=P_{t h}, n=1,2, \ldots, N$. 
Proof: Denote

$$
\begin{aligned}
U_{n}\left(k_{n}, \varepsilon_{n}\right)= & \operatorname{Pr}\left(\mathcal{H}_{n}^{0}\right)\left(1-P_{n}^{f}\left(k_{n}, \varepsilon_{n}\right)\right) R_{n}^{0} \\
& +\operatorname{Pr}\left(\mathcal{H}_{n}^{1}\right)\left(1-P_{n}^{d}\left(k_{n}, \varepsilon_{n}\right)\right) R_{n}^{1}
\end{aligned}
$$

and therefore we have $U\left(\left\{k_{n}\right\},\left\{\varepsilon_{n}\right\}\right)=\sum_{n=1}^{N} U_{n}\left(k_{n}, \varepsilon_{n}\right)$.

From equations (11) and (12), it can be seen that both $\left(1-P_{n}^{f}\left(k_{n}, \varepsilon_{n}\right)\right)$ and $\left(1-P_{n}^{d}\left(k_{n}, \varepsilon_{n}\right)\right)$ in $U_{n}\left(k_{n}, \varepsilon_{n}\right)$ grow with the increase of $\varepsilon_{n}$. On the other hand, the term $\left(1-P_{n}^{d}\left(k_{n}, \varepsilon_{n}\right)\right)$ should be bounded by $1-P_{t h}$. Therefore, $U_{n}\left(k_{n}, \varepsilon_{n}\right)$ achieves its maximal value when $\left(1-P_{n}^{d}\left(k_{n}, \varepsilon_{n}\right)\right)$ reaches its upper bound $\left(1-P_{t h}\right)$, which happens when $P_{n}^{d}\left(k_{n}, \varepsilon_{n}\right)=P_{t h}$.

This completes the proof.

Define

$$
S\left(\left\{k_{n}\right\}\right)=\sum_{n=1}^{N} \operatorname{Pr}\left(\mathcal{H}_{n}^{0}\right)\left(1-P_{n}^{f}\left(k_{n}, P_{n}^{d}=P_{t h}\right)\right) R_{n}^{0}
$$

where $P_{n}^{f}\left(k_{n}, P_{n}^{d}\right)$ is from (7), i.e.,

$$
P_{n}^{f}\left(k_{n}, P_{n}^{d}\right)=Q\left(\left(\gamma_{n}+1\right) Q^{-1}\left(P_{n}^{d}\right)+\gamma_{n} \sqrt{\mu k_{n} \delta}\right) .
$$

Based on Lemma 2, we substitute $P_{n}^{d}$ with $P_{t h}$ in the objective function $U\left(\left\{k_{n}\right\},\left\{\varepsilon_{n}\right\}\right)$ in problem $\mathrm{P} 2$, and we have

$$
\begin{aligned}
& \left.U\left(\left\{k_{n}\right\},\left\{\varepsilon_{n}\right\}\right)\right|_{P_{n}^{d}\left(k_{n}, \varepsilon_{n}\right)=P_{\text {th }}} \\
& =S\left(\left\{k_{n}\right\}\right)+\sum_{n=1}^{N} \operatorname{Pr}\left(\mathcal{H}_{n}^{1}\right)\left(1-P_{t h}\right) R_{n}^{1}
\end{aligned}
$$

in which the second term on the right-hand side of the equality is a fixed value.

Consider the constraints in problem P2. From equation (11), the constraint $\varepsilon_{n} \leq \sigma^{2}\left(1+\gamma_{n}\right)$ in problem P2 corresponds to the constraint $P_{n}^{d} \geq 0.5$, which can be guaranteed by setting $P_{n}^{d}=P_{t h}>0.5$. From equation (12), the constraint $\varepsilon_{n} \geq \sigma^{2}$ in problem $\mathrm{P} 2$ corresponds to the constraint $P_{n}^{f} \leq 0.5$, which can be expressed similarly to equation (8) as

$$
\left(\gamma_{n}+1\right) Q^{-1}\left(P_{t h}\right)+\gamma_{n} \sqrt{\mu k_{n} \delta} \geq 0, \quad n=1,2, \ldots, N
$$

and is equivalent to

$$
k_{n} \geq\left(\frac{-\left(\gamma_{n}+1\right) Q^{-1}\left(P_{t h}\right)}{\sqrt{\delta \mu} \gamma_{n}}\right)^{2}, \quad n=1,2, \ldots, N .
$$

Then by defining $z_{n}=\left[\left(\frac{-\left(\gamma_{n}+1\right) Q^{-1}\left(P_{t h}\right)}{\sqrt{\delta \mu} \gamma_{n}}\right)^{2}\right]$ (where $\lceil\cdot\rceil$ is the ceiling function) and $q_{n}=k_{n}-z_{n}(n=1,2, \ldots, N)$, and rewriting $S\left(\left\{k_{n}\right\}\right)$ in the form of $S\left(\left\{q_{n}\right\}\right)$, problem P2 is equivalent to the following problem.

Problem P3 (with a specific $k$ value)

$$
\begin{array}{ll}
\max _{\left\{q_{n}\right\}} & S\left(\left\{q_{n}\right\}\right)= \\
& \sum_{n=1}^{N} \operatorname{Pr}\left(\mathcal{H}_{n}^{0}\right)\left(1-P_{n}^{f}\left(q_{n}, P_{n}^{d}=P_{t h}\right)\right) R_{n}^{0} \\
& \sum_{n=1}^{N} q_{n}=k M-\sum_{n=1}^{N} z_{n} \\
& q_{n} \geq 0, q_{n}+1 \in \mathcal{I}, \quad n=1,2, \ldots, N \\
& k \in \mathcal{I}
\end{array}
$$

in which

$$
\begin{aligned}
& P_{n}^{f}\left(q_{n}, P_{n}^{d}=P_{t h}\right) \\
& \quad=Q\left(\left(\gamma_{n}+1\right) Q^{-1}\left(P_{t h}\right)+\sqrt{\left(q_{n}+z_{n}\right) \delta \mu} \gamma_{n}\right) .
\end{aligned}
$$

Note that to make the constraints in problem P3 feasible, $k$ should satisfy $k \geq\left\lceil\frac{\sum_{n=1}^{N} z_{n}}{M}\right\rceil$.

The following lemma is in order.

Lemma 3: The function $S\left(\left\{q_{n}\right\}\right)$ in problem P3 is an increasing concave function with respect to $q_{n}(n=1,2, \ldots, N)$ within the region $\sum_{n=1}^{N} q_{n}=k M-\sum_{n=1}^{N} z_{n}, q_{n} \geq 0(n=$ $1,2, \ldots, N)$.

Proof: The first order derivative of $S\left(\left\{q_{n}\right\}\right)$ over $q_{n}$ is

$$
\begin{aligned}
& \frac{\partial S\left(\left\{q_{n}\right\}\right)}{\partial q_{n}} \\
= & -\operatorname{Pr}\left(\mathcal{H}_{n}^{0}\right) R_{n}^{0} \cdot \frac{\partial Q\left(\left(\gamma_{n}+1\right) Q^{-1}\left(P_{t h}\right)+\sqrt{\delta\left(q_{n}+z_{n}\right) \mu} \gamma_{n}\right)}{\partial q_{n}} \\
= & \frac{\operatorname{Pr}\left(\mathcal{H}_{n}^{0}\right) R_{n}^{0}}{2 \sqrt{2 \pi}} e^{-\frac{\left(\left(\gamma_{n}+1\right) Q^{-1}\left(P_{t h}\right)+\sqrt{\delta\left(q_{n}+z_{n}\right) \mu} \gamma_{n}\right)^{2}}{2}} \sqrt{\frac{\mu \delta}{\left(q_{n}+z_{n}\right)}} \gamma_{n} \\
> & 0 .
\end{aligned}
$$

So $S\left(\left\{q_{n}\right\}\right)$ is an increasing function with respect to $q_{n}$.

We define $s_{n}\left(q_{n}\right) \triangleq \operatorname{Pr}\left(\mathcal{H}_{n}^{0}\right)\left(1-P_{n}^{f}\left(q_{n}, P_{n}^{d}=P_{t h}\right)\right) R_{n}^{0}$. Therefore, $S\left(\left\{q_{n}\right\}\right)=\sum_{n=1}^{N} s_{n}\left(q_{n}\right)$. The second order derivative of $s_{n}\left(q_{n}\right)$ over $q_{n}$ is given in (28) on the next page. Note that the inequality in the derivation in (28) is based on $\left(\left(\gamma_{n}+1\right) Q^{-1}\left(P_{t h}\right)+\sqrt{\delta\left(q_{n}+z_{n}\right) \mu} \gamma_{n}\right) \geq 0$, which is from (23) based on the fact that $P_{n}^{f} \leq 0.5$.

Define $\boldsymbol{q}^{a} \triangleq\left(q_{1}^{a}, q_{2}^{a}, \ldots, q_{N}^{a}\right)$ and $\boldsymbol{q}^{b} \triangleq\left(q_{1}^{b}, q_{2}^{b}, \ldots, q_{N}^{b}\right)$ such that

$$
\begin{aligned}
& q_{n}^{a} \geq 0, q_{n}^{b} \geq 0,1 \leq n \leq N \\
& \sum_{n=1}^{N} q_{n}^{a}=\sum_{n=1}^{N} q_{n}^{b}=k M-\sum_{n=1}^{N} z_{n} .
\end{aligned}
$$

For any $\lambda \in[0,1]$, it is easy to see that $\boldsymbol{q}^{c} \triangleq \lambda \boldsymbol{q}^{a}+(1-\lambda) \boldsymbol{q}^{b}$ satisfies

$$
q_{n}^{c} \geq 0,1 \leq n \leq N ; \quad \sum_{n=1}^{N} q_{n}^{c}=k M-\sum_{n=1}^{N} z_{n} .
$$

So in the region $\sum_{n=1}^{N} q_{n}=k M-\sum_{n=1}^{N} z_{n}, q_{n} \geq 0 \quad(n=$ $1, \ldots, N)$, we have

$$
\begin{aligned}
S\left(\left\{q_{n}^{c}\right\}\right) & =\sum_{n=1}^{N} s_{n}\left(q_{n}^{c}\right) \\
& =\sum_{n=1}^{N} s_{n}\left(\lambda q_{n}^{a}+(1-\lambda) q_{n}^{b}\right) \\
\text { from }(28) & \sum_{n=1}^{N} \lambda s_{n}\left(q_{n}^{a}\right)+\sum_{n=1}^{N}(1-\lambda) s_{n}\left(q_{n}^{b}\right) \\
= & \lambda S\left(\left\{q_{n}^{a}\right\}\right)+(1-\lambda) S\left(\left\{q_{n}^{b}\right\}\right)
\end{aligned}
$$

which means that the function $S\left(\left\{q_{n}\right\}\right)$ is concave within the region $\sum_{n=1}^{N} q_{n}=k M-\sum_{n=1}^{N} z_{n}, q_{n} \geq 0(n=1, \ldots, N)$. 


$$
\begin{aligned}
\frac{\partial^{2} s_{n}\left(q_{n}\right)}{\partial q_{n}{ }^{2}}= & -\frac{\operatorname{Pr}\left(\mathcal{H}_{n}^{0}\right) R_{n}^{0}}{4 \sqrt{2 \pi}} \cdot\left(e^{-\frac{\left(\left(\gamma_{n}+1\right) Q^{-1}\left(P_{t h}\right)+\sqrt{\delta\left(q_{n}+z_{n}\right) \mu} \gamma_{n}\right)^{2}}{2}} \sqrt{\frac{\mu \delta}{\left(q_{n}+z_{n}\right)^{3}}} \gamma_{n}\right. \\
& \left.+e^{-\frac{\left(\left(\gamma_{n}+1\right) Q^{-1}\left(P_{t h}\right)+\sqrt{\delta\left(q_{n}+z_{n}\right) \mu} \gamma_{n}\right)^{2}}{2}} \cdot \frac{\mu \delta}{\left(q_{n}+z_{n}\right)} \gamma_{n}^{2} \cdot\left(\left(\gamma_{n}+1\right) Q^{-1}\left(P_{t h}\right)+\sqrt{\delta\left(q_{n}+z_{n}\right) \mu} \gamma_{n}\right)\right) \\
< & 0 .
\end{aligned}
$$

This completes the proof.

From Lemma 3, problem P3 becomes a convex mixedinteger problem. Generally a mixed-integer problem is NPhard [14]. However, for problem P3, thanks to the separability and concavity of the objective function and the linear constraints with variable coefficients all being 1's, an incremental algorithm [15, page 384] can be used to solve problem P3 with polynomial complexity $O(k M \log N+N)$, which can converge to the global optimal point as proved in reference [16, pages 53-54].

The procedure of the incremental algorithm for problem P3, referred to as Algorithm 1, is as follows.

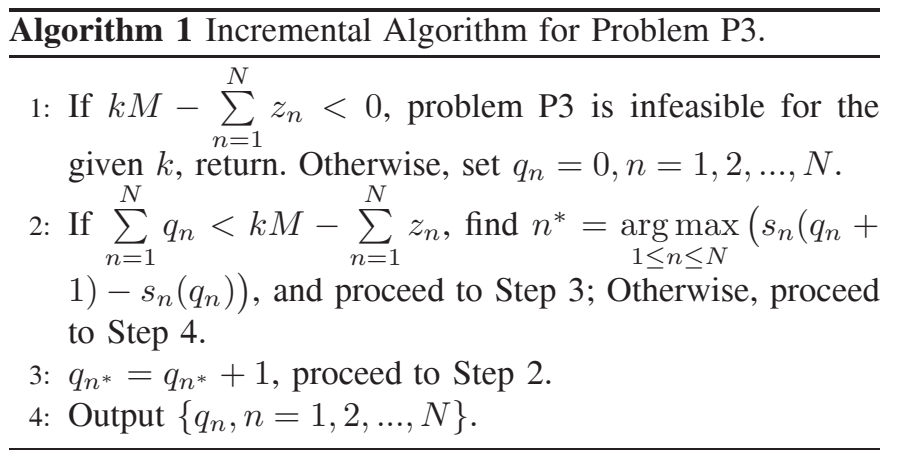

Since problems P2 and P3 are equivalent, the optimal objective value of problem $\mathrm{P} 2$ (with a specific $k$ value), i.e., $U^{*}(k)$, can be obtained by setting $k_{n}=q_{n}+z_{n}$ and $P_{n}^{d}\left(k_{n}, \varepsilon_{n}\right)=P_{t h}, n=1,2, \ldots, N$.

Lemma 4: $U^{*}(k)$ has two properties: (1) $U^{*}(k)$ is an increasing function; (2) $U^{*}(k)-U^{*}(k-1) \geq U^{*}(k+1)-U^{*}(k)$ for $k \geq\left\lceil\frac{\sum_{n=1}^{N} z_{n}}{M}\right\rceil, k \in \mathcal{I}$.

The proof of Lemma 4 is given in the Appendix.

\section{Optimal Solution to Problem P1}

In Section III-B, we have provided an optimal solution to problem P2, which is a subproblem of problem P1. Now, we can proceed to solve problem $\mathrm{P} 1$. With the solution of problem $\mathrm{P} 2$, denoted $U^{*}(k)$ for a specific $k$, problem $\mathrm{P} 1$ is equivalent to

$$
\begin{array}{ll}
\max _{k} & C(k)=\frac{T-k \delta}{T} \cdot U^{*}(k) \\
\text { s.t. } & {\left[\frac{\sum_{n=1}^{N} z_{n}}{M}\right] \leq k \leq\left\lfloor\frac{T}{\delta}\right\rfloor, k \in \mathcal{I} .}
\end{array}
$$

We have the following lemma for the objective function of this problem.

$$
\begin{aligned}
& \text { Lemma 5: } C(k+1)-C(k)<C(k)-C(k-1) \text {, for } \\
& \left\lceil\frac{\sum_{n=1}^{N} z_{n}}{M}\right\rceil<k<\left\lfloor\frac{T}{\delta}\right\rfloor, k \in \mathcal{I} .
\end{aligned}
$$

$$
\begin{aligned}
\text { Proof: } & {[C(k+1)-C(k)]-[C(k)-C(k-1)] } \\
= & {\left[\left(1-\frac{(k+1) \delta}{T}\right) U^{*}(k+1)-\left(1-\frac{k \delta}{T}\right) U^{*}(k)\right] } \\
& -\left[\left(1-\frac{k \delta}{T}\right) U^{*}(k)-\left(1-\frac{(k-1) \delta}{T}\right) U^{*}(k-1)\right] \\
= & \left(1-\frac{k \delta}{T}\right)\left[\left(U^{*}(k+1)-U^{*}(k)\right)-\right. \\
& \left.\left(U^{*}(k)-U^{*}(k-1)\right)\right]+\frac{\delta}{T}\left(U^{*}(k-1)-U^{*}(k+1)\right) .
\end{aligned}
$$

From Lemma 4, $U^{*}(k+1)-U^{*}(k) \leq U^{*}(k)-U^{*}(k-1)$, and $U^{*}(k-1)<U^{*}(k+1)$. Thus we have

$$
[C(k+1)-C(k)]-[C(k)-C(k-1)]<0 .
$$

This completes the proof.

From Lemma 5, we see that as $k$ grows, the increase of $C(k)$, denoted $D(k)=C(k)-C(k-1)$, becomes smaller. Then, the optimal point of $k$, denoted $k^{*}$, satisfies

$$
D\left(k^{*}\right) \geq 0 \geq D\left(k^{*}+1\right) .
$$

In the search for $k^{*}$, for a round (with a specific $k$ value), the results in previous rounds are still useful, and can be used to reduce the computation complexity in the current round. And the total complexity is upper bounded by $O\left(\left\lfloor\frac{T}{\delta}\right\rfloor M \log N+N\right)$.

\section{Optimal Sensing Time Setting in the Continuous-Time Sensing Mode}

In this section, we consider the continuous-time sensing mode. The duration of the sensing phase in a slot is denoted $\tau$. So the total sensing time among all the secondary users is $M \tau$. The total sensing time assigned to channel $n \in\{1,2, \ldots, N\}$ is $\tau_{n}$. Note that $\tau$ and $\tau_{n}$ 's are all continuous variables. Similar to problem P1, we have the following problem formulation.

\section{Problem P4 :}

$$
\begin{array}{cl}
\max _{\tau,\left\{\tau_{n}\right\},\left\{\varepsilon_{n}\right\}} & C_{c}=\left(1-\frac{\tau}{T}\right) \\
& \\
& \quad\left(\sum _ { n = 1 } ^ { N } \left(\operatorname{Pr}\left(\mathcal{H}_{n}^{0}\right)\left(1-P_{n}^{f}\left(\tau_{n}, \varepsilon_{n}\right)\right) R_{n}^{0}\right.\right. \\
& \left.\left.\quad+\operatorname{Pr}\left(\mathcal{H}_{n}^{1}\right)\left(1-P_{n}^{d}\left(\tau_{n}, \varepsilon_{n}\right)\right) R_{n}^{1}\right)\right) \\
\text { s.t. } & P_{n}^{d}\left(\tau_{n}, \varepsilon_{n}\right) \geq P_{t h}, \quad n=1,2, \ldots, N \\
& \sigma^{2} \leq \varepsilon_{n} \leq \sigma^{2}\left(1+\gamma_{n}\right), \quad n=1,2, \ldots, N \\
& \sum_{n=1}^{N} \tau_{n}=\tau M \\
& 0<\tau \leq T \\
& \tau_{n}>0, \quad n=1,2, \ldots, N
\end{array}
$$

in which $P_{n}^{f}\left(\tau_{n}, \varepsilon_{n}\right)$ and $P_{n}^{d}\left(\tau_{n}, \varepsilon_{n}\right)$ are given in (5) and (4), respectively. 
Similar to Lemma 1, it can be proved that problem P4 is not a convex problem. In order to solve it, we transform problem P4 to:

$$
\begin{array}{cl}
\max _{\tau} & C_{c}(\tau)=\left(1-\frac{\tau}{T}\right) \cdot U_{c}^{*}(\tau) \\
\text { s.t. } & 0<\tau \leq T .
\end{array}
$$

where $U_{c}^{*}(\tau)$ is the optimal objective value of the following problem with a specific $\tau$ value:

$$
\begin{array}{cl}
\underset{\left\{\tau_{n}\right\},\left\{\varepsilon_{n}\right\}}{\text { Problem P5 }} & \text { (with a specific } \tau \text { value) } \\
& U_{c}\left(\left\{\tau_{n}\right\},\left\{\varepsilon_{n}\right\}\right)= \\
& \sum_{n=1}^{N}\left(\operatorname{Pr}\left(\mathcal{H}_{n}^{0}\right)\left(1-P_{n}^{f}\left(\tau_{n}, \varepsilon_{n}\right)\right) R_{n}^{0}\right. \\
& \left.\quad+\operatorname{Pr}\left(\mathcal{H}_{n}^{1}\right)\left(1-P_{n}^{d}\left(\tau_{n}, \varepsilon_{n}\right)\right) R_{n}^{1}\right) \\
\text { s.t. } & P_{n}^{d}\left(\tau_{n}, \varepsilon_{n}\right) \geq P_{t h}, \quad n=1,2, \ldots, N \\
& \sigma^{2} \leq \varepsilon_{n} \leq \sigma^{2}\left(1+\gamma_{n}\right), \quad n=1,2, \ldots, N \\
& \sum_{n=1}^{N} \tau_{n}=\tau M \\
& \tau_{n}>0, \quad n=1,2, \ldots, N .
\end{array}
$$

Similar to the proof of Lemma 2, it can be proved that function $U_{c}\left(\left\{\tau_{n}\right\},\left\{\varepsilon_{n}\right\}\right)$ achieves the maximal value when $P_{n}^{d}\left(\tau_{n}, \varepsilon_{n}\right)=P_{t h}$.

Define

$$
S_{c}\left(\left\{\tau_{n}\right\}\right)=\sum_{n=1}^{N} \operatorname{Pr}\left(\mathcal{H}_{n}^{0}\right)\left(1-P_{n}^{f}\left(\tau_{n}, P_{n}^{d}=P_{t h}\right)\right) R_{n}^{0}
$$

where $P_{n}^{f}\left(\tau_{n}, P_{n}^{d}\right)$ is from (7), i.e.,

$$
P_{n}^{f}\left(\tau_{n}, P_{n}^{d}\right)=Q\left(\left(\gamma_{n}+1\right) Q^{-1}\left(P_{n}^{d}\right)+\gamma_{n} \sqrt{\mu \tau_{n}}\right) .
$$

We substitute $P_{n}^{d}\left(\tau_{n}, \varepsilon_{n}\right)$ with $P_{t h}$ in the objective function $U_{c}\left(\left\{\tau_{n}\right\},\left\{\varepsilon_{n}\right\}\right)$ in problem P5, and we have

$$
\begin{aligned}
& \left.U_{c}\left(\left\{\tau_{n}\right\},\left\{\varepsilon_{n}\right\}\right)\right|_{P_{n}^{d}\left(\tau_{n}, \varepsilon_{n}\right)=P_{t h}} \\
& =S_{c}\left(\left\{\tau_{n}\right\}\right)+\sum_{n=1}^{N} \operatorname{Pr}\left(\mathcal{H}_{n}^{1}\right)\left(1-P_{t h}\right) R_{n}^{1}
\end{aligned}
$$

in which the second term on the right-hand side of the equality is a fixed value.

Similar to the discussion in Section III, the constraint $\varepsilon_{n} \leq$ $\sigma^{2}\left(1+\gamma_{n}\right)$ in problem P5 is satisfied when $P_{n}^{d}=P_{t h}>0.5$, and the constraint $\varepsilon_{n} \geq \sigma^{2}$ is equivalent to the following inequality

$$
\left(\gamma_{n}+1\right) Q^{-1}\left(P_{t h}\right)+\gamma_{n} \sqrt{\mu \tau_{n}} \geq 0, \quad n=1,2, \ldots, N
$$

which means

$$
\tau_{n} \geq\left(\frac{-\left(\gamma_{n}+1\right) Q^{-1}\left(P_{t h}\right)}{\sqrt{\mu} \gamma_{n}}\right)^{2}, \quad n=1,2, \ldots, N .
$$

Denote $z_{n}^{c}=\left(\frac{-\left(\gamma_{n}+1\right) Q^{-1}\left(P_{t h}\right)}{\sqrt{\mu} \gamma_{n}}\right)^{2}$. Then problem P5 is equivalent to the following problem.

$$
\begin{array}{cl}
\underset{\left\{\tau_{n}\right\}}{\text { Problem P6 }} & \text { (with a specific } \tau \text { value) } \\
& S_{c}\left(\left\{\tau_{n}\right\}\right)= \\
& \sum_{n=1}^{N}\left(\operatorname{Pr}\left(\mathcal{H}_{n}^{0}\right)\left(1-P_{n}^{f}\left(\tau_{n}, P_{n}^{d}=P_{t h}\right)\right) R_{n}^{0}\right) \\
\text { s.t. } & \sum_{n=1}^{N} \tau_{n}=\tau M \\
& \tau_{n} \geq z_{n}^{c}, \quad n=1,2, \ldots, N .
\end{array}
$$

It can be easily proved that in the feasible region of problem P6 (in which $P_{n}^{f} \leq 0.5$ is guaranteed), the objective function of problem P6 is a concave function, and therefore, problem P6 is a convex problem.

Let $S_{c}^{*}(\tau)$ denote the optimal objective value of problem P6 with a specific $\tau$ value.

Lemma 6: Function $S_{c}^{*}(\tau)$ is an increasing concave function for $\tau \geq \frac{\sum_{n=1}^{N} z_{n}^{c}}{M}$.

Proof: We first prove it is an increasing function.

Define a function

$$
s_{n}^{c}\left(\tau_{n}\right)=\operatorname{Pr}\left(\mathcal{H}_{n}^{0}\right)\left(1-P_{n}^{f}\left(\tau_{n}, P_{n}^{d}=P_{t h}\right)\right) R_{n}^{0}, \quad \tau_{n} \geq z_{n}^{c} .
$$

It is easy to prove that function $s_{n}^{c}\left(\tau_{n}\right)$ is an increasing function.

For any two variables $\tau^{\dagger} \geq \frac{\sum_{n=1}^{N} z_{n}^{c}}{M}$ and $\tau^{\ddagger} \geq \frac{\sum_{n=1}^{N} z_{n}^{c}}{M}$, assume that $\tau^{\dagger}<\tau^{\ddagger}$. For $\tau=\tau^{\dagger}$, the optimal solution to problem P6 is denoted $\left\{\tau_{n}^{\dagger}\right\}$, with the optimal objective value being $S_{c}^{*}\left(\tau^{\dagger}\right)$. For $\tau=\tau^{\ddagger}$, the optimal solution to problem P6 is denoted $\left\{\tau_{n}^{\ddagger}\right\}$, with the optimal objective value being $S_{c}^{*}\left(\tau^{\ddagger}\right)$. Let $\tau_{N}^{\prime}=\tau^{\ddagger} M-\sum_{i=1}^{N-1} \tau_{n}^{\dagger}$. Since $\sum_{i=1}^{N} \tau_{n}^{\dagger}=\tau^{\dagger} M$, we have

$$
\tau_{N}^{\prime}=\tau^{\ddagger} M-\left(\tau^{\dagger} M-\tau_{N}^{\dagger}\right)=\left(\tau^{\ddagger}-\tau^{\dagger}\right) M+\tau_{N}^{\dagger}>\tau_{N}^{\dagger} .
$$

Since $s_{N}^{c}\left(\tau_{N}\right)$ is an increasing function, we have $s_{N}^{c}\left(\tau_{N}^{\prime}\right)>$ $s_{N}^{c}\left(\tau_{N}^{\dagger}\right)$, and further we have

$$
S_{c}^{*}\left(\tau^{\dagger}\right)=\sum_{i=1}^{N-1} s_{i}^{c}\left(\tau_{i}^{\dagger}\right)+s_{N}^{c}\left(\tau_{N}^{\dagger}\right)<\sum_{i=1}^{N-1} s_{i}^{c}\left(\tau_{i}^{\dagger}\right)+s_{N}^{c}\left(\tau_{N}^{\prime}\right) .
$$

On the other hand, $\left\{\tau_{1}^{\dagger}, \ldots, \tau_{N-1}^{\dagger}, \tau_{N}^{\prime}\right\}$ is also a feasible solution for problem P6 with $\tau=\tau^{\ddagger}$. Recall that $S_{c}^{*}\left(\tau^{\ddagger}\right)$ is the optimal objective value of problem P6 when $\tau=\tau^{\ddagger}$. So we have

$$
S_{c}^{*}\left(\tau^{\ddagger}\right) \geq \sum_{i=1}^{N-1} s_{i}^{c}\left(\tau_{i}^{\dagger}\right)+s_{N}^{c}\left(\tau_{N}^{\prime}\right) .
$$

From (40) and (41), we have $S_{c}^{*}\left(\tau^{\dagger}\right)<S_{c}^{*}\left(\tau^{\ddagger}\right)$, which means that $S_{c}^{*}(\tau)$ is an increasing function.

Next we prove that $S_{c}^{*}(\tau)$ is a concave function. Denote a vector $\boldsymbol{\nu}=\left(\tau_{1}, \tau_{2}, \ldots, \tau_{N}\right)$, and two functions

$$
H_{t c}(\boldsymbol{\nu})=\sum_{n=1}^{N}\left(\operatorname{Pr}\left(\mathcal{H}_{n}^{0}\right)\left(1-P_{n}^{f}\left(\tau_{n}, P_{n}^{d}=P_{t h}\right)\right) R_{n}^{0}\right)
$$

and

$$
H_{c}(x)=\max _{\boldsymbol{\nu} \in \mathcal{C}(x)} H_{t c}(\boldsymbol{\nu})
$$

where $\mathcal{C}(x)=\left\{\left(\tau_{1}, \tau_{2}, \ldots, \tau_{N}\right) \mid \sum_{n=1}^{N} \tau_{n}=x, \tau_{n} \geq z_{n}^{c}, n=\right.$ $1,2, \ldots, N\}$. It is obvious that $H_{t c}^{n=1}(\boldsymbol{\nu})$ is a concave function with respect to $\boldsymbol{\nu}$ when $\tau_{n} \geq z_{n}^{c}, n=1,2, \ldots, N$.

For any two values $x_{1}, x_{2}>0$, and any $\epsilon>0$, there exist $\boldsymbol{\nu}_{\mathbf{1}} \in \mathcal{C}\left(x_{1}\right)$ and $\boldsymbol{\nu}_{\mathbf{2}} \in \mathcal{C}\left(x_{2}\right)$ such that $H_{t c}\left(\boldsymbol{\nu}_{\mathbf{1}}\right) \geq H_{c}\left(x_{1}\right)-\epsilon$ 
and $H_{t c}\left(\boldsymbol{\nu}_{\mathbf{2}}\right) \geq H_{c}\left(x_{2}\right)-\epsilon$. For any $\theta \in[0,1]$, we have

$$
\begin{aligned}
H_{c}\left(\theta x_{1}+(1-\theta) x_{2}\right) & =\max _{\boldsymbol{\nu} \in \mathcal{C}\left(\theta x_{1}+(1-\theta) x_{2}\right)} H_{t c}(\boldsymbol{\nu}) \\
& \geq H_{t c}\left(\theta \boldsymbol{\nu}_{\mathbf{1}}+(1-\theta) \boldsymbol{\nu}_{\mathbf{2}}\right) \\
& >\theta H_{t c}\left(\boldsymbol{\nu}_{\mathbf{1}}\right)+(1-\theta) H_{t c}\left(\boldsymbol{\nu}_{\mathbf{2}}\right) \\
& \geq \theta H_{c}\left(x_{1}\right)+(1-\theta) H_{c}\left(x_{2}\right)-\epsilon .
\end{aligned}
$$

This inequality holds for any small $\epsilon>0$. So we have

$$
H_{c}\left(\theta x_{1}+(1-\theta) x_{2}\right)>\theta H_{c}\left(x_{1}\right)+(1-\theta) H_{c}\left(x_{2}\right)
$$

which means $H_{c}(x)$ is a concave function. And therefore, $S_{c}^{*}(\tau)=H_{c}(\tau M)$ is also a concave function [17, page 84] .

This completes the proof.

With the optimal objective value of problem P5, i.e., $U_{c}^{*}(\tau)$, problem P4 is equivalent to the problem in (34) with constraint $\frac{\sum_{n=1}^{N} z_{n}^{c}}{M} \leq \tau \leq T .^{2}$ And the objective function $C_{c}(\tau)$ of the problem in (34) has the following property.

Lemma 7: $C_{c}(\tau)$ is a concave function when $\tau \geq \frac{\sum_{n=1}^{N} z_{n}^{c}}{M}$.

Proof: The second-order derivative of $C_{c}(\tau)$ is given by

$$
\frac{d^{2} C_{c}(\tau)}{d \tau^{2}}=\frac{d^{2} S_{c}^{*}(\tau)}{d \tau^{2}}\left(1-\frac{\tau}{T}\right)+2 \cdot\left(-\frac{1}{T}\right) \frac{d S_{c}^{*}(\tau)}{d \tau} .
$$

Since function $S_{c}^{*}(\tau)$ is increasing and concave (from Lemma 6), we have $\frac{d S_{c}^{*}(\tau)}{d \tau}>0$ and $\frac{d^{2} S_{c}^{*}(\tau)}{d \tau^{2}}<0$. Therefore, $\frac{d^{2} C_{c}(\tau)}{d \tau^{2}}<$ 0 , which means that function $C_{c}(\tau)$ is a concave function.

From Lemma 7, it can be seen that, problem P4 is transformed to a bilevel optimization problem [15], where the upper level problem (i.e., the problem in (34), which optimizes $\tau$ ) and lower level problem (i.e., problem P5 or P6, which optimizes $\left\{\tau_{n}\right\}$ ) are both convex problems. The bilevel problem can be solved by existing methods.

\section{Performance Evaluation}

In this section, numerical results are presented to verify the effectiveness of our proposed algorithms. Similar to [12], the system setup is as follows. The sampling rate is $\mu=6 \mathrm{MHz}$; the slot duration is $T=100 \mathrm{~ms}$; the threshold of detection probability is $P_{t h}=0.9$; and $S N R_{n}^{s s}$ between secondary users are all assumed to have a mean $20 \mathrm{~dB}$. Both $S N R_{n}^{p s}$ (the SNR from the primary user to a secondary user on channel $n$ ) and $S N R_{n}^{s s}$ are assumed to be exponentially distributed.

\section{A. Verification of Our Algorithms}

The slotted-time sensing mode is tested first. Consider $N=$ 2 channels, with the available probability as $\operatorname{Pr}\left(\mathcal{H}_{1}^{0}\right)=0.8$ and $\operatorname{Pr}\left(\mathcal{H}_{2}^{0}\right)=0.6$, and mean SNR from primary user to both secondary users as $\gamma_{1}=-15 \mathrm{~dB}$ in channel 1 and $\gamma_{2}=-20$ $\mathrm{dB}$ in channel 2 . There are $M=2$ secondary users. The minislot duration is $\delta=0.1 \mathrm{~ms}$. To get the value of $C(k)$ (given in (19)) at each $k$, we use two methods: through exhaustive search of the $k_{n}$ 's and $\varepsilon_{n}$ 's, and through our Algorithm 1. The results are demonstrated in Fig. 3. It is obvious that the

\footnotetext{
${ }^{2}$ Note that when $0<\tau<\frac{\sum_{n=1}^{N} z_{n}^{c}}{M}$, other constraints in the original problem, P4, cannot be satisfied simultaneously.
}

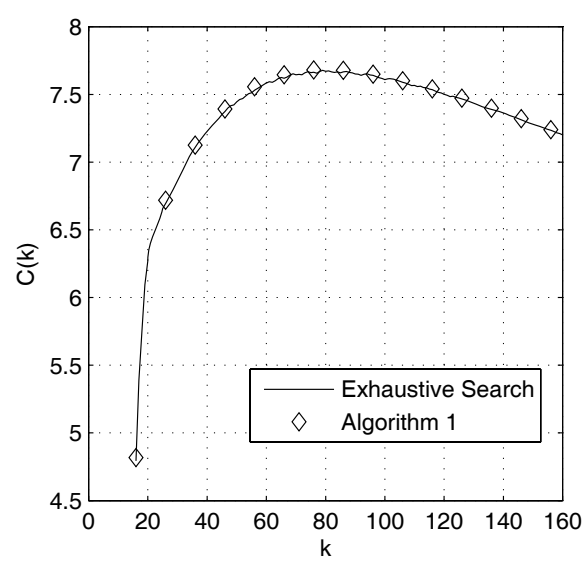

Fig. 3. $C(k)$ versus $k$.

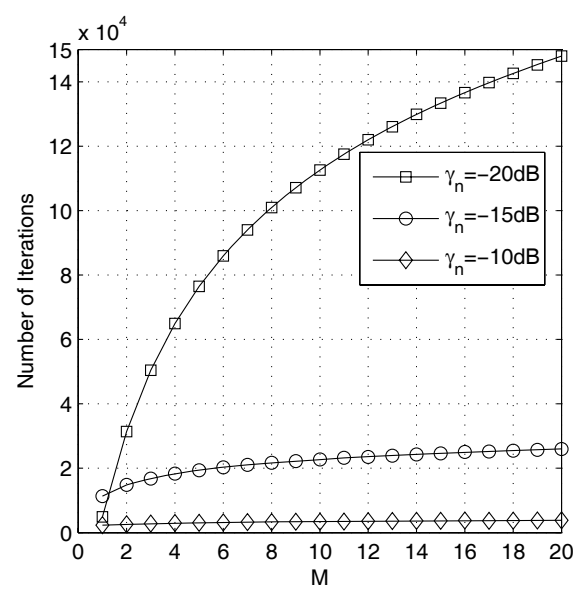

Fig. 4. Number of iterations versus $M(N=10, \delta=0.01 \mathrm{~ms})$.

results match well with each other. And the concave-shaped $C(k)$ is also consistent with the conclusion in Lemma 5. All these verify the correctness of our algorithm.

For continuous-time sensing mode, similar observations are noticed, and the results are omitted here.

\section{B. Complexity of Algorithm 1}

Next, we illustrate the complexity of Algorithm 1 for slotted-time sensing mode, when the mini-slot duration is 0.01 ms. The number of iterations to reach the optimal solution is taken as the measure of complexity. Fig. 4 shows the complexity when the number of channels, $N$, is fixed as 10, while Fig. 5 shows the complexity when the number of secondary users, $M$, is fixed as 10 .

\section{Comparison between the Slotted-time Sensing Mode and Continuous-time Sensing Mode}

To compare the performance between slotted-time sensing mode and continuous-time sensing mode, consider $N=5$ channels. These 5 channels have available probabilities as $0.8,0.7,0.6,0.5$, and 0.4 , with $\gamma_{n}$ being $-19 \mathrm{~dB},-18 \mathrm{~dB}$, $-17 \mathrm{~dB},-16 \mathrm{~dB}$, and $-15 \mathrm{~dB}$, respectively. By running our 


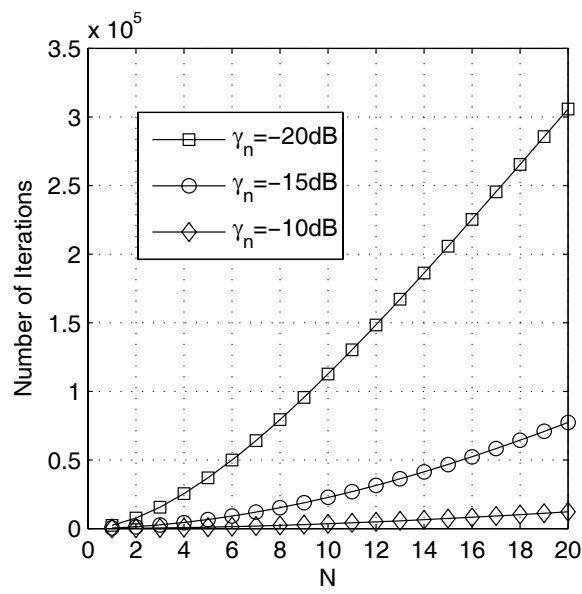

Fig. 5. Number of iterations versus $N(M=10, \delta=0.01 \mathrm{~ms})$.

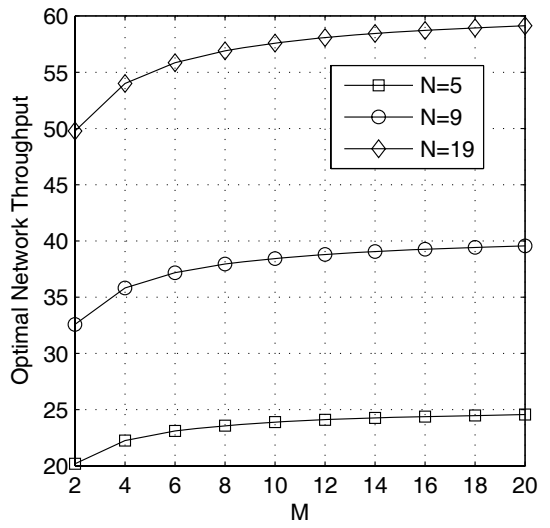

Fig. 6. Optimal network throughput versus $M$ and $N$.

algorithms for the continuous-time sensing mode, and slottedtime sensing mode with different mini-slot duration $\delta=0.01$ $\mathrm{ms}, 0.05 \mathrm{~ms}, 0.1 \mathrm{~ms}, 0.5 \mathrm{~ms}$, or $1 \mathrm{~ms}$, the obtained optimal network throughput is 17.4 in all cases.

In comparison of slotted-time sensing mode and continuoustime sensing mode, it is difficult to tell which one is better. Generally, it is easier to implement the slotted-time sensing mode in a real system. On the other hand, the complexity in solving the problem with continuous-time sensing mode is lower than that with the slotted-time sensing mode.

Since both sensing modes can reach almost the same optimal network throughput, in the following, we evaluate the performance of slotted-time sensing mode only.

\section{Impact of the Number of Channels and the Number of Users}

In the system setup, there are $N=5,9$, or 19 channels. Channel $n(1 \leq n \leq N)$ has a free probability $\operatorname{Pr}\left(\mathcal{H}_{n}^{0}\right)=$ $1-0.05 * n$, and the average channel gain from the primary user to a secondary user is $\gamma_{n}=(-20+n-1)$ dB. Fig. 6 shows the optimal network throughput (obtained from our algorithm) as a function of the number, $M$, of secondary users, with different $N$ values. It can be seen that when the number of channels or the number of secondary users increases, the optimal network throughput also increases. Interestingly, with a fixed $N$ value, when the value of $M$ further increases beyond a certain value, the optimal network throughput seems to keep almost constant. The reason is as follows. When $M$ increases, the total sensing time for the channels also increases, which means that a smaller false alarm probability for each channel is expected. When $M$ is large enough, the false alarm probability for each channel is almost zero. Recall that the optimal objective function of problem P1 is achieved when the detection probability is exactly the threshold $P_{t h}$ (from Lemma 2). Therefore, when the false alarm probability of each channel is almost zero, the optimal objective function in problem $\mathrm{P} 1$ can be approximated as $\sum_{n=1}^{N}\left(\operatorname{Pr}\left(\mathcal{H}_{n}^{0}\right) R_{n}^{0}+\operatorname{Pr}\left(\mathcal{H}_{n}^{1}\right)\left(1-P_{t h}\right) R_{n}^{1}\right)$, which is a constant. Note that in the approximation, we omit the factor $\frac{T-k \delta}{T}$, since the sensing duration is only a small portion of the total duration $T$.

\section{CONCLUSIONS}

In this paper, we have explored the optimal multi-channel cooperative spectrum sensing strategies in cognitive radio networks. We have studied the problem of how to determine the total sensing time and how to distribute the total sensing time to different channels in cooperative soft-decision spectrum sensing. For the slotted-time sensing mode, we have transformed the initial nonconvex mixed-integer problem into convex mixed-integer subproblems, and provided a polynomialcomplexity algorithm to achieve the optimal solution of the initial problem. For the continuous-time sensing mode, we have successfully transformed the initial nonconvex optimization problem into a convex bilevel optimization problem. This research should provide helpful insights into the sensing time configuration in cognitive radio networks.

In this research, it is assumed that a secondary user can send its test statistic to the coordinator. An interesting research topic is to investigate the case when a secondary user sends a quantized version of its test statistic to the coordinator or sends its detection decision on the presence or absence of primary activities (i.e., 1-bit information is sent to the coordinator). It is also interesting to study the problem from a game theoretical point of view, in which each secondary user is assumed to be selfish but rational.

In our problem formulation, the constraint $P_{n}^{f} \leq 0.5$ may lead to some performance loss. For instance, for a specific channel (say channel $n$ ), consider the case that the channel gains from the primary user to secondary users and from the secondary transmitter assigned in channel $n$ to its receiver are significantly lower than the channel gains in other channels. Then channel $n$ will dominate the sensing time, but the return of secondary throughout in this channel is very low. A possible solution is to sense only a subset of the $N$ channels. However, the selection of the subset depends on the channel SNR values and the channel available probabilities. How to find an optimal subset of channels is an interesting research topic for further investigation.

\section{APPEndix: Proof of Lemma 4}

Proof: In Algorithm 1, Step 2 and Step 3 are repeated by $\left(k M-\sum_{n=1}^{N} z_{n}\right)$ times, referred to as $\left(k M-\sum_{n=1}^{N} z_{n}\right)$ 
rounds. After round $i$ is completed, we denote the value of $q_{n}$ as $q_{n}^{(i)}, n=1,2, \ldots, N$. Then Algorithm 1 can be represented equivalently in an alternative way:

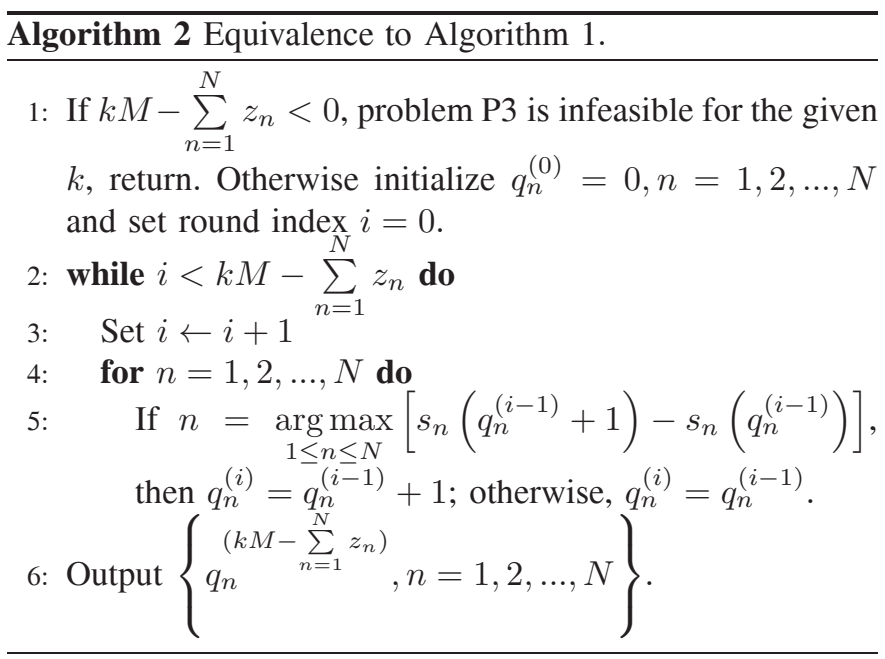

We denote the channel selected in round $i$ as $c(i)$ :

$$
c(i)=\underset{1 \leq n \leq N}{\arg \max }\left[s_{n}\left(q_{n}^{(i-1)}+1\right)-s_{n}\left(q_{n}^{(i-1)}\right)\right] .
$$

If we record the value of $\left.U\left(\left\{k_{n}\right\},\left\{\varepsilon_{n}\right\}\right)\right|_{P_{n}^{d}\left(k_{n}, \varepsilon_{n}\right)=P_{t h}}$ as

$$
\begin{aligned}
& \left.U\left(\left\{k_{n}\right\},\left\{\varepsilon_{n}\right\}\right)\right|_{P_{n}^{d}\left(k_{n}, \varepsilon_{n}\right)=P_{t h}} \stackrel{q_{n}=k_{n}-z_{n}}{=} \\
& \sum_{n=1}^{N}\left(\operatorname{Pr}\left(\mathcal{H}_{n}^{0}\right)\left(1-P_{n}^{f}\left(q_{n}, P_{t h}\right)\right) R_{n}^{0}+\operatorname{Pr}\left(\mathcal{H}_{n}^{1}\right)\left(1-P_{t h}\right) R_{n}^{1}\right)
\end{aligned}
$$

after each round, totally we have $\left(k M-\sum_{n=1}^{N} z_{n}\right)$ values, denoted $H(1), H(2), \ldots, H\left(k M-\sum_{n=1}^{N} z_{n}\right)$, respectively. Here $P_{n}^{f}\left(q_{n}, P_{t h}\right)$ is given in (26).

Recall that Algorithm 1 leads to an optimal solution of problem P2. So the optimal objective value of problem P2 (with a specific $k$ ), i.e., $U^{*}(k)$, is given by

$$
U^{*}(k)=H\left(k M-\sum_{n=1}^{N} z_{n}\right) .
$$

In (47), the second term in the summation on the right-hand side of the equality is a fixed value. So we have

$$
\begin{aligned}
& H(i)-H(i-1) \\
= & \operatorname{Pr}\left(\mathcal{H}_{c(i)}^{0}\right)\left(1-P_{c(i)}^{f}\left(q_{c(i)}^{(i)}, P_{t h}\right)\right) R_{c(i)}^{0} \\
& -\operatorname{Pr}\left(\mathcal{H}_{c(i)}^{0}\right)\left(1-P_{c(i)}^{f}\left(q_{c(i)}^{(i-1)}, P_{t h}\right)\right) R_{c(i)}^{0} \\
= & s_{c(i)}\left(q_{c(i)}^{(i)}\right)-s_{c(i)}\left(q_{c(i)}^{(i)}-1\right) .
\end{aligned}
$$

We first prove property (1): $U^{*}(k)$ is an increasing function.

Similar to (27) and (28), it can be proved that $s_{n}\left(q_{n}\right)$ is an increasing and concave function with respect to $q_{n}$. So we have

$$
s_{c(i)}\left(q_{c(i)}^{(i)}\right)-s_{c(i)}\left(q_{c(i)}^{(i)}-1\right)>0
$$

and thus

$$
H(i)-H(i-1)>0, \quad i>1 .
$$

This means $H(i)$ is an increasing function. Further, based on (48), we have

$$
\begin{aligned}
& U^{*}(k)-U^{*}(k-1) \\
& =H\left(k M-\sum_{n=1}^{N} z_{n}\right)-H\left((k-1) M-\sum_{n=1}^{N} z_{n}\right) \\
& >0 .
\end{aligned}
$$

This means that $U^{*}(k)$ is an increasing function.

Now we proceed to prove property (2) of $U^{*}(k)$. We first compare $[H(i)-H(i-1)]$ with $[H(i+1)-H(i)]$. The difference is given by

$$
\begin{aligned}
& {[H(i)-H(i-1)]-[H(i+1)-H(i)]} \\
& =\left[s_{c(i)}\left(q_{c(i)}^{(i)}\right)-s_{c(i)}\left(q_{c(i)}^{(i)}-1\right)\right] \\
& \quad-\left[s_{c(i+1)}\left(q_{c(i+1)}^{(i+1)}\right)-s_{c(i+1)}\left(q_{c(i+1)}^{(i+1)}-1\right)\right] .
\end{aligned}
$$

We have two scenarios as follows.

- If $c(i)=c(i+1)=j$ : We have $q_{j}^{(i+1)}=q_{j}^{(i)}+1$. From (50), we have

$$
\begin{aligned}
& {[H(i)-H(i-1)]-[H(i+1)-H(i)]} \\
& =\left[s_{j}\left(q_{j}^{(i)}\right)-s_{j}\left(q_{j}^{(i)}-1\right)\right] \\
& \quad-\left[s_{j}\left(q_{j}^{(i)}+1\right)-s_{j}\left(q_{j}^{(i)}\right)\right] \\
& \quad \stackrel{(a)}{>} 0
\end{aligned}
$$

where $(a)$ follows from the fact that $s_{n}\left(q_{n}\right)$ is a concave function with respect to $q_{n}$.

- If $c(i) \neq c(i+1)$ : In round $i, c(i)=$ $\underset{1 \leq n \leq N}{\arg \max }\left[s_{n}\left(q_{n}^{(i-1)}+1\right)-s_{n}\left(q_{n}^{(i-1)}\right)\right]$. This means that

$$
\begin{aligned}
& s_{c(i)}\left(q_{c(i)}^{(i-1)}+1\right)-s_{c(i)}\left(q_{c(i)}^{(i-1)}\right) \\
& \geq s_{c(i+1)}\left(q_{c(i+1)}^{(i-1)}+1\right)-s_{c(i+1)}\left(q_{c(i+1)}^{(i-1)}\right) .
\end{aligned}
$$

Since $q_{c(i)}^{(i)}=q_{c(i)}^{(i-1)}+1$, we have

$$
\begin{aligned}
s_{c(i)}\left(q_{c(i)}^{(i-1)}\right. & +1)-s_{c(i)}\left(q_{c(i)}^{(i-1)}\right) \\
& =s_{c(i)}\left(q_{c(i)}^{(i)}\right)-s_{c(i)}\left(q_{c(i)}^{(i)}-1\right) .
\end{aligned}
$$

Since $q_{c(i+1)}^{(i+1)}=q_{c(i+1)}^{(i)}+1=q_{c(i+1)}^{(i-1)}+1$, we have

$$
\begin{aligned}
& s_{c(i+1)}\left(q_{c(i+1)}^{(i-1)}+1\right)-s_{c(i+1)}\left(q_{c(i+1)}^{(i-1)}\right) \\
& \quad=s_{c(i+1)}\left(q_{c(i+1)}^{(i+1)}\right)-s_{c(i+1)}\left(q_{c(i+1)}^{(i+1)}-1\right) .
\end{aligned}
$$

From (50) - (53), it can be seen that

$$
[H(i)-H(i-1)]-[H(i+1)-H(i)] \geq 0 .
$$

Therefore, in either of the above scenarios, we always have

$$
[H(i)-H(i-1)]-[H(i+1)-H(i)] \geq 0, \quad i>1
$$

and it follows that

$H(i)-H(i-1) \geq H(i+M)-H(i+M-1), \quad i>1$. 
Then, we have

$$
\begin{aligned}
& U^{*}(k)-U^{*}(k-1) \\
& =H\left(k M-\sum_{n=1}^{N} z_{n}\right)-H\left((k-1) M-\sum_{n=1}^{N} z_{n}\right) \\
& =\sum_{i=(k-1) M-\sum_{n=1}^{N} z_{n}+1}^{k M-\sum_{n=1}^{N} z_{n}}(H(i)-H(i-1)) \\
& \text { from }(54)^{(54)} \sum_{i=k M-\sum_{n=1}^{N} z_{n}+1}^{(k+1) M-\sum_{n=1}^{N} z_{n}}(H(i)-H(i-1)) \\
& =U^{*}(k+1)-U^{*}(k) .
\end{aligned}
$$

This completes the proof.

\section{ACKNOWLEDGMENTS}

The authors would like to thank the Editor Dr. Rohit Nabar and the anonymous reviewers for their constructive comments and suggestions which helped to improve the quality of the manuscript.

\section{REFERENCES}

[1] G. Staple and K. Werbach, "The end of spectrum scarcity," IEEE Spectrum, vol. 41, pp. 48-52, Mar. 2004.

[2] Federal Communications Commission, "Spectrum policy task force report, FCC 02-155," Nov. 2002.

[3] S. Haykin, "Cognitive radio: brain-empowered wireless communications," IEEE J. Sel. Areas Commun., vol. 23, no. 2, pp. 201-220, Feb. 2005 .

[4] S. M. Kay, Fundamentals of Statistical Signal Processing, Volume 2: Detection Theory. Prentice Hall, 1998.

[5] G. Ganesan and Y. Li, "Cooperative spectrum sensing in cognitive radio-part I: two user networks," IEEE Trans. Wireless Commun., vol. 6, no. 6, pp. 2204-2213, June 2007.

[6] G. Ganesan and Y. Li, "Cooperative spectrum sensing in cognitive radio-part II: multiuser networks," IEEE Trans. Wireless Commun., vol. 6, no. 6, pp. 2214-2222, June 2007.

[7] M. Gandetto and C. Regazzoni, "Spectrum sensing: a distributed approach for cognitive terminals," IEEE J. Sel. Areas Commun., vol. 25, no. 3, pp. 546-557, Apr. 2007

[8] Q. Zhao, L. Tong, A. Swami, and Y. Chen, "Decentralized cognitive MAC for opportunistic spectrum access in ad hoc networks: a POMDP framework," IEEE J. Sel. Areas Commun., vol. 25, no. 3, pp. 589-600, Apr. 2007.

[9] H. Jiang, L. Lai, R. Fan, and H. V. Poor, "Optimal selection of channel sensing order in cognitive radio," IEEE Trans. Wireless Commun., vol. 8, no. 1, pp. 297-307, Jan. 2009.
[10] L. Lai, H. El Gamal, H. Jiang, and H. V. Poor, "Cognitive medium access: exploration, exploitation and competition," accepted with minor revision to IEEE Trans. Mobile Comput.

[11] R. Fan and H. Jiang, "Channel sensing-order setting in cognitive radio networks: a two-user case," IEEE Trans. Veh. Technol., vol. 58, no. 9, pp. 4997-5008, Nov. 2009.

[12] Y.-C. Liang, Y. Zeng, E. C. Y. Peh, and A. T. Hoang, "Sensingthroughput tradeoff for cognitive radio networks," IEEE Trans. Wireless Commun., vol. 7, no. 4, pp. 1326-1337, Apr. 2008.

[13] X. Kang, Y.-C. Liang, H. K. Garg, and L. Zhang, "Sensing-based spectrum sharing in cognitive radio networks," IEEE Trans. Veh. Technol., vol. 58, no. 8, pp. 4649-4654, Oct. 2009.

[14] M. R. Bussieck and A. Pruessner, "Mixedinteger nonlinear programming." [Online]. Available: http://www.gamsworld.org/minlp/siagopt.pdf.

[15] C. Floudas and P. M. Pardalos, Encyclopedia of Optimization. Springer Press, 2nd edition, 2009.

[16] T. Ibaraki and N. Katoh, Resource Allocation Problems: Algorithmic Approaches. The MIT Press, 1988.

[17] S. P. Boyd and L. Vandenberghe, Convex Optimization. Cambridge University Press, 2004.

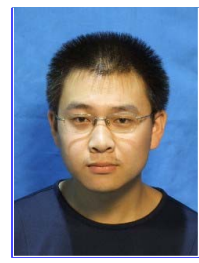

Rongfei Fan received the B.E. degree in 2007 in electrical engineering from Harbin Institute of Technology, China. He is currently working toward his Ph.D. degree at the Department of Electrical and Computer Engineering, University of Alberta, Canada. His research interests include cognitive radio networking and cross-layer design.

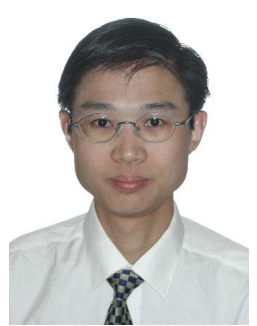

Hai Jiang (M'07) received the B.Sc. and M.Sc. degrees in electronics engineering from Peking University, Beijing, China, in 1995 and 1998, respectively, and the Ph.D. degree (with an Outstanding Achievement in Graduate Studies Award) in electrical engineering from the University of Waterloo, Waterloo, Ontario, Canada, in 2006.

Since July 2007, he has been an Assistant Professor with the Department of Electrical and Computer Engineering, University of Alberta, Edmonton, Alberta, Canada. His research interests include radio resource management, cognitive radio networking, and cross-layer design for wireless multimedia communications.

Dr. Jiang is an Associate Editor for the IEEE TRAnsactions ON VEHICular TeChNOLOGY. He served as a Co-Chair for the General Symposium at the International Wireless Communications and Mobile Computing Conference in 2007, the Communications and Networking Symposium at the Canadian Conference on Electrical and Computer Engineering in 2009, and the Wireless and Mobile Networking Symposium at the IEEE International Conference on Communications in 2010. He received an Alberta Ingenuity New Faculty Award in 2008 and a Best Paper Award from the IEEE Global Communications Conference (GLOBECOM) in 2008. 